\title{
Entwicklungen im Bereich der Fernerkundung für forstliche Anwendungen
}

Christian Ginzler

Lars T. Waser
Landschaftsdynamik, Eidgenössische Forschungsanstalt für Wald, Schnee und Landschaft (CH)*

Landschaftsdynamik, Eidgenössische Forschungsanstalt für Wald, Schnee und Landschaft (CH)

\section{Entwicklungen im Bereich der Fernerkundung für forstliche Anwendungen}

\begin{abstract}
In den letzten zehn Jahren sind im Bereich von Methoden und Daten der Fernerkundung für forstliche Anwendungen grosse Fortschritte erzielt worden. Viele Datensätze stehen nicht mehr nur für Fallstudien und Methodenentwicklungen zur Verfügung, sondern auch für die grossflächige Anwendung, und sie werden mittlerweile zum Teil auch in kurzen Abständen aktualisiert. Luftbilder, die auch das nahe Infrarot erfassen, werden heute dank den digitalen Kameras routinemässig aufgenommen. Farbinfrarot-Orthobilder lassen sich auf kantonaler und nationaler Ebene sehr einfach in die GIS-Anwendung einbinden. Daten aus flugzeuggetragenem Laserscanning sind für viele Anwendungen fast schon ein Standard geworden. Es existieren in die gewohnte Arbeitsumgebung integrierbare Werkzeuge, die es erlauben, mit den 3-D-Punktwolken Analysen sehr effizient durchzuführen und nützliche Produkte für den Waldbereich zu realisieren. Auch terrestrisches Laserscanning steht kurz vor der operationellen Anwendbarkeit. Fernerkundung entwickelt sich immer mehr zu einem Werkzeug für die praktische, tägliche Arbeit. Ist man sich der Möglichkeiten, aber auch der Grenzen bewusst, so vermag die Fernerkundung substanzielle Beiträge zur Unterstützung und Optimierung von terrestrischen Inventuren zu leisten und grossflächig Mehrwerte zu generieren.
\end{abstract}

Keywords: airborne laser scanning ALS, terrestrial laser scanning TLS, image matching, inventories, multispectral data

doi: $10.3188 /$ szf. 2017.0118

* Zürcherstrasse 111, CH-8903 Birmensdorf, E-Mail christian.ginzler@wsl.ch

$\mathrm{D}$ er Einsatz der Fernerkundung hat im Wald eine sehr lange Tradition. Bereits im letzten Jahrhundert wurden Luftbilder für die Erfassung und Beschreibung von Waldbeständen eingesetzt. Die Anwendungen waren jedoch lange auf Kartierungen, Interpretationen und qualitative Ansprachen beschränkt (Oester 1992, Scherrer et al 1990). Ende der 1970er-Jahre wurden Satellitenbilder (räumliche Auflösung maximal $30 \mathrm{~m}$ ) verfügbar, der Schritt zur praktischen Anwendung war aber langsam. Erst in den letzten 20 Jahren, durch die stetige Digitalisierung, die Effizienzsteigerung bei der Datenprozessierung und die schnellere Verfügbarkeit der Daten aus der Erdbeobachtung, setzten sich Methoden und Daten aus der Fernerkundung im praktischen Alltag nach und nach durch. Für die praktischen forstlichen Anwendungen in der Schweiz sind insbesondere jene Sensoren interessant, die sich in den letzten Jahren hinsichtlich Handhabung, technischer Eigenschaften, Zuverlässigkeit und somit auch Kontinuität etablieren konnten.
Welche forstlichen Kenngrössen lassen sich mit der Fernerkundung erfassen? Zu unterscheiden sind zwei Ebenen: Auf der Ebene des Einzelbaums können Höhe, Kronenbreite, Baumart und Vitalität direkt gemessen werden. Merkmale, die von oben nicht direkt messbar sind, können modelliert (z.B. der Brusthöhendurchmesser [BHD] über die Beziehung zu Baumart und Höhe) oder mittels terrestrischer Fernerkundung gemessen werden. Auf der Ebene des Bestands oder der Behandlungseinheit können Merkmale wie mittlere Höhen, Kronenschluss, vertikale und horizontale Strukturen und Baumtypenanteile bestimmt werden. Für nicht direkt bestimmbare Merkmale wie Vorrat oder Biomasse sind Modelle entwickelt worden, um sie mit Fernerkundungsdaten indirekt zu ermitteln. Viele Merkmale können mit unterschiedlichen Sensoren bestimmt werden. So können Baumtypen mit spektralen Daten (Abbildungen 1 und 2), aber auch mit flugzeuggetragenem Laserscanning bestimmt werden (Hollaus \& Pfeifer 2017, dieses Heft). 


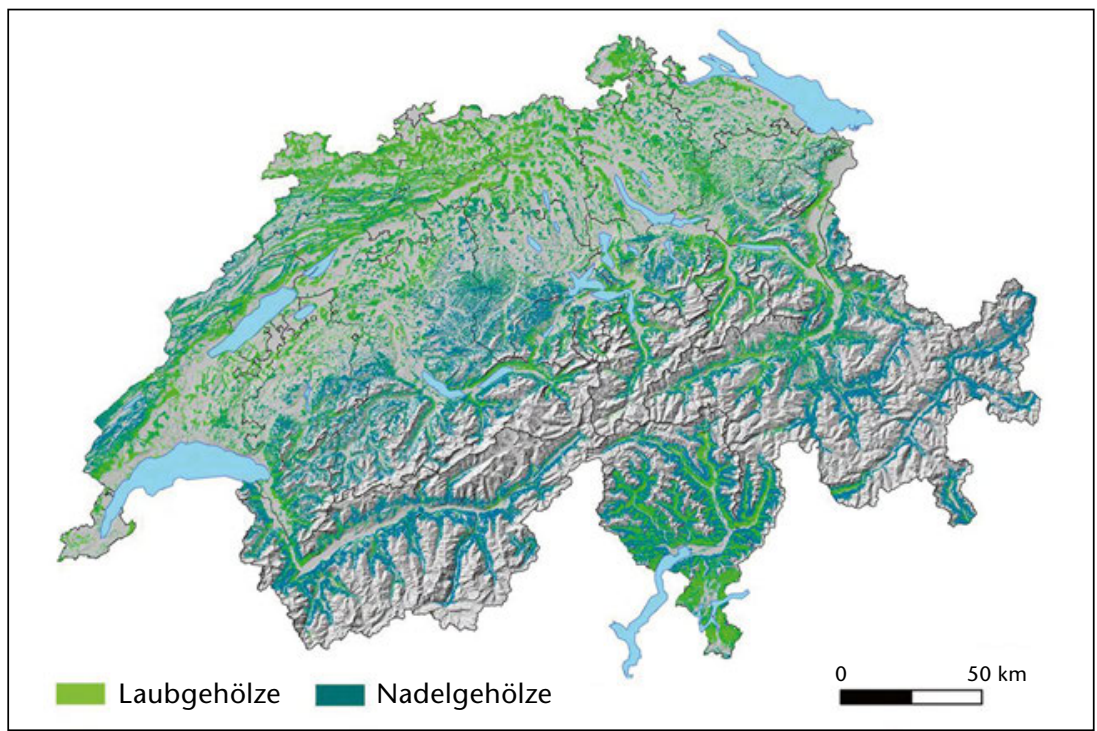

Abb 1 Unterscheidung von Laub- und Nadelgehölzen der Schweiz basierend auf den ADS80-Luftbilddaten der Swisstopo. @ Landesforstinventar

Im Folgenden stellen wir die Fortschritte bei den Fernerkundungsmethoden verschiedener Systeme und Sensoren im Zusammenhang mit forstlichen Fragestellungen dar. Wir zeigen einerseits die Bereiche, wo der Schritt in die praktische Anwendung bereits erfolgt ist, und andererseits solche, wo wir mittelfristig ein grosses Potenzial für Praxisanwendungen sehen.

\section{Spektrale Fernerkundung}

\section{Multispektrale Aufnahmen}

Kameras und multispektrale Sensoren zeichnen das von der Erdoberfläche reflektierte Sonnenlicht auf und werden daher auch passive Systeme genannt. Im letzten Jahrhundert genutzt wurden vor allem Schwarz-Weiss-Luftbilder - für die quantitative Erfassung von Wald, die Abgrenzung von Wald und Nichtwald und die Unterteilung der Wälder in homogene Bestandeseinheiten. Ab den 1970er-Jahren kamen Farbinfrarot (FIR)-Luftbilder für die qualitative Charakterisierung von Bäumen und Waldbeständen hinzu (Oester 1992, Gillis \& Leckie 1996). Durch die Sichtbarmachung des nahen Infrarots konnten Baumarten und die Vitalität von Bäumen und Beständen interpretiert werden. FIR-Luftbilder bilden auch heute noch die operationelle Datengrundlage für die Erstellung von Bestandeskarten.

Die Verfügbarkeit von FIR-Luftbildern hat in den letzten zehn Jahren deutlich zugenommen. Vor den digitalen Luftbildkameras waren Spezialbefliegungen mit analogem FIR-Filmmaterial nötig. Dies war aufwendig und teuer, da der Markt für FIR-Filme und deren Aufnahmen beschränkt war. Flächendeckende FIR-Luftbilddaten existierten somit nicht. Seit dem operationellen Einsatz von grossformatigen digitalen Luftbildkameras wird in vier Kanälen neben den sichtbaren Farbkanälen (rot, grün, blau) auch das nahe Infrarot standardmässig aufgezeichnet. Durch die regelmässigen Befliegungsprogramme auf nationaler und kantonaler Ebene stehen landesweit immer sehr aktuelle FIR-Luftbilder zur Verfügung.

Seit 2008 sind für die Schweiz flächendeckend digitale Stereoluftbilder vom Zeilensensor ADS80 mit einer räumlichen Auflösung von 25 bis $50 \mathrm{~cm}$ und den Kanälen Rot, Grün, Blau und nahes Infrarot vorhanden, die im 6-Jahres-Rhythmus jeweils während der Vegetationsperiode (Mai-September) aufgenommen werden. Dazu kommen digitale Luftbilddaten, welche im Rahmen von kantonalen Befliegungsprogrammen akquiriert werden. Diese Da-
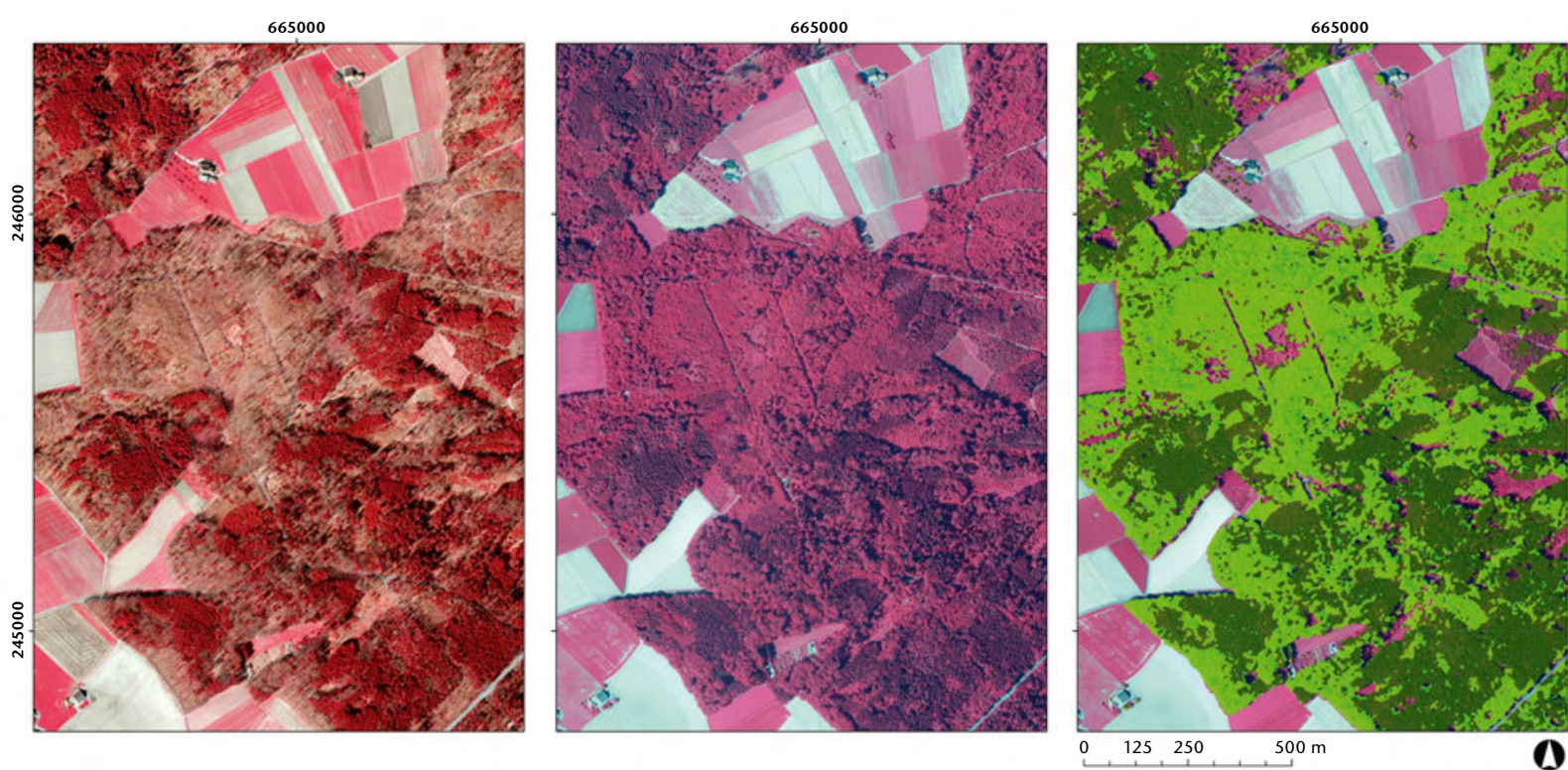

Abb 2 Unterscheidung von Laub- und Nadelgehölzen der Schweiz basierend auf jährlichen Luftbilddaten im Kanton Aargau. Links: FIR-Orthobild vom Frühjahr 2012, Mitte: FIR-Orthobild vom Sommer 2013, rechts: Klassifikation in Laub- und Nadelgehölze basierend auf den Vegetationsindizes aus den beiden FIR-Orthobildern. Abbildungen links und Mitte: ๑ Kanton Aargau 


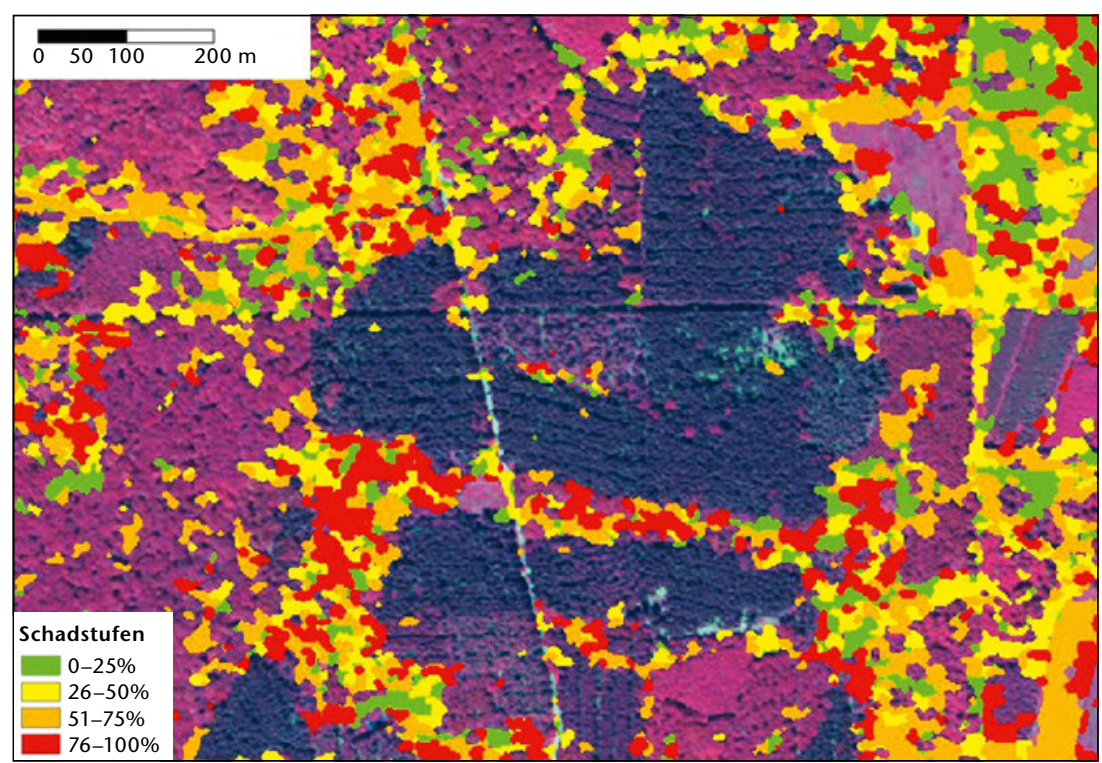

Abb 3 Erfassung des Ausmasses des Eschentriebsterbens in Mecklenburg-Vorpommern in vier Stufen mittels 8-Kanal-WorldView-2-Satellitenbildern (Waser 2014).

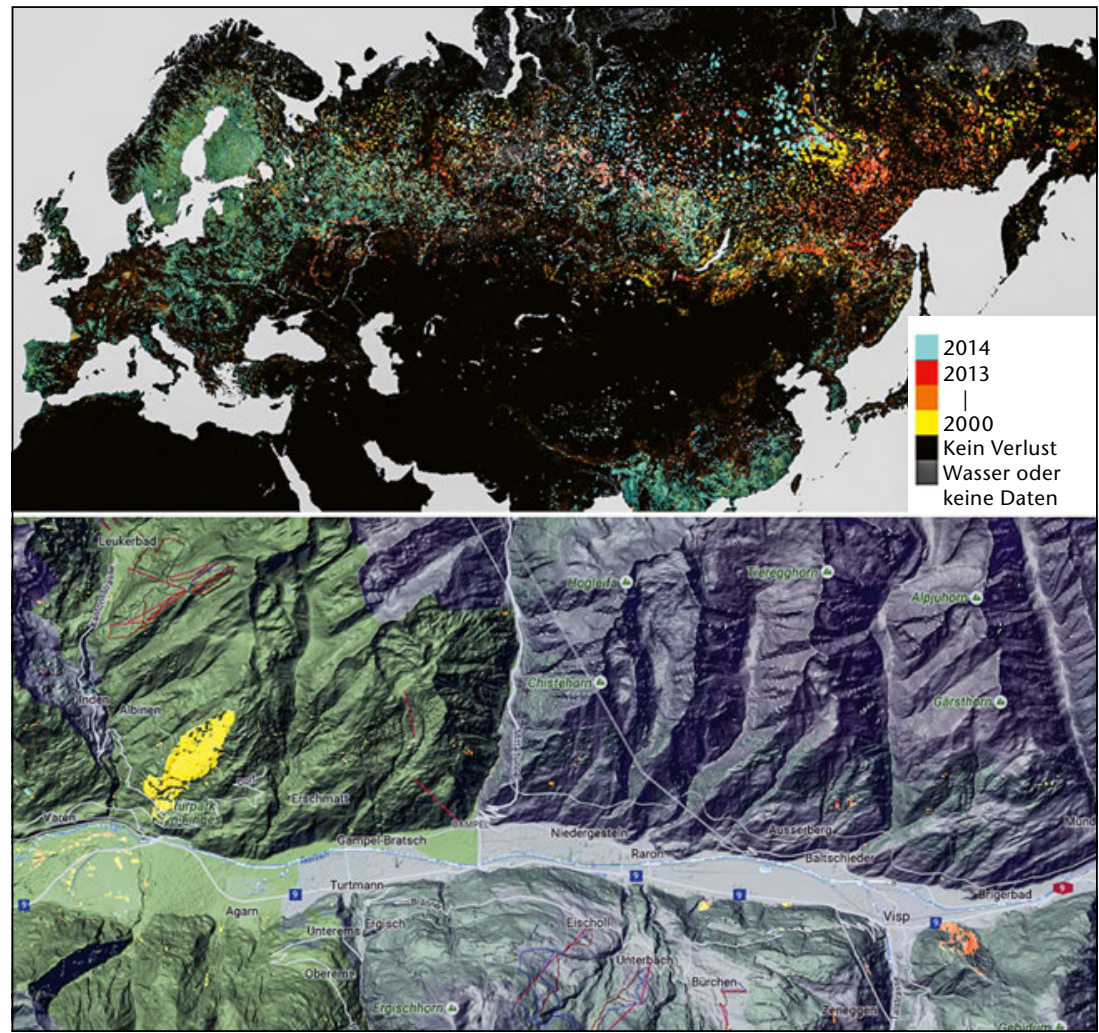

Abb 4 Erfassung von Waldveränderungen (hier: Abnahme der bestockten Flächen zwischen 2000 und 2014 in jährlicher Auflösung im globalen, freien Datensatz «Global Forest Change». Die Analyse basiert auf Landsat-Daten. Oben: Ausschnitt Eurasien mit starker Waldnutzung im Osten Russlands. Unten: Detailausschnitt des Wallis mit den gut sichtbaren Waldbränden von Leuk (2003) und Visp (2012).

ten bilden die Grundlage für flächige Produkte auf kantonaler und nationaler Ebene, die im Rahmen des Landesfortinventars (LFI) entwickelt werden. Basierend auf den 4-Kanal-ADS80-Luftbildstreifen der Swisstopo wird derzeit ein neuer, hochaufgelöster Datensatz zum Waldmischungsgrad der Schweiz erstellt (Abbildung 1). Das Verfahren ist bezüglich Datenvorverarbeitung, Klassifikation, Validierung und Erstellung der Karte automatisiert.
Eine weitere Möglichkeit, Laub- und Nadelgehölze zu differenzieren, ist der Vergleich von Luftbildern, die im Frühjahr und im Sommer aufgenommen worden sind. In Kantonen mit jährlich abwechselnder Befliegungszeit kann so sehr einfach ein Laub-/Nadelgehölz-Datensatz gerechnet werden (Abbildung 2).

Auch die ab den 1980er-Jahren aufgekommenen kommerziell genutzten Erdbeobachtungssatelliten ermöglichen das Erstellen von flächendeckenden Datensätzen für den Waldbereich. Die Anwendungsgebiete reichen von der Wald-Nichtwald-Trennung über die Erfassung von Schadenereignissen und die Klassierung von Baumarten bis zur Veränderungsdetektion (Franklin 2001). Seit der Jahrtausendwende haben grosse technische Fortschritte bei den Sensoren, innovative Auswertungsalgorithmen sowie leistungsstärkere Hardware die Entwicklung der Anwendungen im Waldbereich weiter vorangetrieben. So können die Hauptbaumarten auf Ebene Region bereits mit hoher Zuverlässigkeit modelliert werden. Neue Generationen von Satellitensensoren wie WorldView-2/-3/-4, GeoEye-1 und Quickbird bieten eine räumliche Auflösung der Schwarz-Weiss-Bänder von mittlerweile unter $30 \mathrm{~cm}$. Die je nach Sensor vier bis acht Multispektralbänder werden mit einer Auflösung von unter $2 \mathrm{~m}$ aufgezeichnet und können mit den Schwarz-Weiss-Bändern zu sehr hoch aufgelösten Multispektralbildern kombiniert werden. Damit können Baumarten, je nach verwendetem Verfahren mit unterschiedlicher Genauigkeit, bestimmt werden (Immitzer et al 2012). Ein Überblick über Bildmaterial und Verfahren zur Baumartenklassifikation ist in Waser (2013) zu finden. Auch verschiedene Vitalitätszustände (Abbildung 3) können erfasst werden (Waser et al 2014).

Die Übertragung der Modelle und Klassifikationen auf andere Gebiete, die nicht in derselben Aufnahme oder Topografie liegen, ist jedoch wegen der topografischen, beleuchtungsspezifischen und phänologischen Einflüsse eine grosse Herausforderung und noch nicht praxisreif gelöst.

\section{Zeitserien von Satellitendaten}

Neben der immer höheren räumlichen Auflösung der Satellitendaten (aktuell $<30 \mathrm{~cm}$ ) wird auch die zeitliche Auflösung immer besser. Die Öffnung der Bildarchive und der freie und sehr einfache $\mathrm{Zu}$ gang zu Satellitendaten (z.B. Landsat-Archiv) hat die Forschungs- und Entwicklungsarbeit stimuliert. Solch lange Zeitreihen haben hohes Potenzial für das Monitoring und die Analyse von Veränderungen, Störungen und Eingriffen auf globaler und auf regionaler Ebene (Abbildung 4). Ein Beispiel ist das Projekt «Global Forest Change», das Veränderungen im Wald mit einer zeitlichen Auflösung von einem Jahr und einer räumlichen Auflösung von $30 \mathrm{~m}$ frei und online zur Verfügung stellt (Hansen et al 2013). 


\section{Bildspektroskopie}

Grosses Potenzial birgt auch die Bildspektroskopie, die Auswertung von hyperspektralen Daten. Im Unterschied zu den multispektralen Sensoren zeichnen hyperspektrale die reflektierte Strahlung der Erdoberfläche kontinuierlich in bis zu hunderten schmalen Kanälen auf. Sie decken den reflektiven Bereich des elektromagnetischen Spektrums vom sichtbaren Licht über Nahinfrarot, Kurzwelleninfrarot bis teilweise Thermalinfrarot ab. Die Bildspektroskopie bietet die Möglichkeit, die biochemische Zusammensetzung der Vegetation zu messen (Huber et al 2008). In Fallstudien wurden Bildspektroskopiedaten zur Klassifikation von Baumarten (Fassnacht et al 2014) oder zur Erfassung von Vitalitätsveränderungen (Lausch et al 2013) eingesetzt. Grossflächige operationelle Anwendung gibt es allerdings noch nicht.

\section{Fernerkundung mit flugzeug- getragenem Laserscanning}

\section{3-D-Aufnahmen von Vegetation und Boden}

Neben den spektralen Informationen der passiven Sensoren haben aktive Sensoren, die ein Lichtsignal aussenden und die Reflexion aufzeichnen, in den letzten Jahren im Waldbereich enorm an Bedeutung gewonnen. Flugzeuggetragenes Laserscanning (Airborne Laser Scanning [ALS], oft auch LiDAR genannt) ist ein solches aktives Aufnahmesystem. Ein Scanner sendet einen Lichtpuls, meist in der Wellenlänge im nahen Infrarotbereich, in Richtung der Bodenoberfläche aus. Der Lichtpuls wird an der Oberfläche reflektiert, und anhand der Zeit zwischen Senden und Empfangen wird die Distanz berechnet. Der grosse Vorteil des aktiven Systems ist, dass bei Vegetationsflächen nicht die gesamte Energie des Lichtpulses an deren Oberfläche reflektiert wird, sondern ein Teil des Strahls in die Vegetation eindringen kann. Im optimalen Fall erreicht der Lichtpuls die Bodenoberfläche und wird dort reflektiert. Der Sensor zeichnet die rückgestrahlte Energie auf. Es können neben der ersten und der letzten Reflexion durch die Analyse der vollen Rückstrahlungsinformation (full-waveform) auch dazwischenliegende Reflexionspeaks ermittelt werden. Somit erhält man mit einem einzigen entsendeten Lichtpuls eine Reihe von diskreten Einzelpunkten. Aus den Reflexionen der Vegetationsoberfläche kann ein digitales Oberflächenmodell (DOM) und aus den Punkten der Bodenoberfläche ein digitales Terrainmodell (DTM) berechnet werden. Aus der Differenz von DOM und DTM kann sehr einfach das sogenannte normalisierte digitale Oberflächenmodell (nDOM) erstellt werden, das die Höhe der Objekte über dem Boden repräsentiert. Werden künstliche Objekte wie Gebäude aus dem nDOM eliminiert, so spricht man von einen digitalen Vegetationshöhenmodell (VHM). Im forstlichen Kontext wird ein VHM oft auch als Kronenhöhenmodell (KHM) bezeichnet.

Neben dem Boden wird die Vegetationsstruktur in den aufgezeichneten Punktwolken sehr detailliert wiedergegeben. Zahlreiche Arbeiten beschäftigten sich mit der Ableitung von Struktur- und Objektparametern aus den Punktwolken (Leiterer et al 2015, Zellweger et al 2016). Für Analysen im Wald kann prinzipiell zwischen flächenbasierten und einzelbaumbasierten Ansätzen unterschieden werden. Beim flächigen Ansatz werden aus den ALS-Daten Parameter extrahiert, um forstlich relevante Merkmale auf der Ebene von Beständen zu modellieren. Aktuelle Arbeiten zeigen, dass Oberhöhe, Vorrat, Grundfläche, Stammzahl und Biomasse sehr gut mit Variablen aus ALS-Punktdaten beschrieben und modelliert werden können (z.B. Hill et al 2014, Straub et al 2010). Vor allem in den skandinavischen Ländern ist eine flächenbasierte Kombination von ALSmit Waldinventur- und Bestandesdaten operationell in Verwendung (Nilsson et al 2016). Je strukturierter und gemischter die Bestände sind, desto schwieriger wird allerdings die Modellierung der forstlichen Merkmale. Bei hoher Punktdichte können Merkmale der Schichtigkeit von Beständen abgeleitet werden (Leiterer et al 2015). Dabei werden die Häufigkeitsverteilungen der ALS-Punkte für definierte Bezugsflächen (z.B. $10 \times 10 \mathrm{~m}$ ) berechnet. Diese Verteilungen erlauben Ableitungen von einund mehrschichtigen Bestandeseinheiten. Die Informationen zur horizontalen und vertikalen Waldstruktur haben sich auch als wertvolle Information erwiesen, um Vorkommen, Verteilung und Häufigkeit von verschiedenen Organismengruppen vorherzusagen (Huber et al 2016; Zellweger \& Bollmann 2017, dieses Heft; Dymytrova et al 2016).

Mit dem einzelbaumbasierten Ansatz wird versucht, aus der Punktwolke oder aus dem gerasterten digitalen KHM die Baumkronen zu detektieren. Viele Methoden existieren bereits, und sie werden stetig weiterentwickelt (Menk et al 2017, dieses Heft).

\section{Stetig zunehmende Punktdichte}

ModerneScanner schicken bis zu 400000 Lichtpulse pro Sekunde, und es kann ein neuer Lichtpuls ausgesendet werden, bevor der letzte detektiert wurde, was das effiziente Erfassen einer sehr hohen Punktdichte am Boden über ein grosses Gebiet erlaubt. So lag die mittlere Punktdichte bei der landesweiten Befliegung der Schweiz von 2000 bis 2008 bei durchschnittlich 0.5 Punkten pro Quadratmeter. Aktuelle Datensätze haben hingegen eine Dichte von 20 Punkten pro Quadratmeter und mehr. Diese Daten erlauben zum Beispiel eine detaillierte Modellierung der Bodenoberfläche auch in bewaldeten Gebieten (Abbildung 5). Die grossen Datenmengen, die 

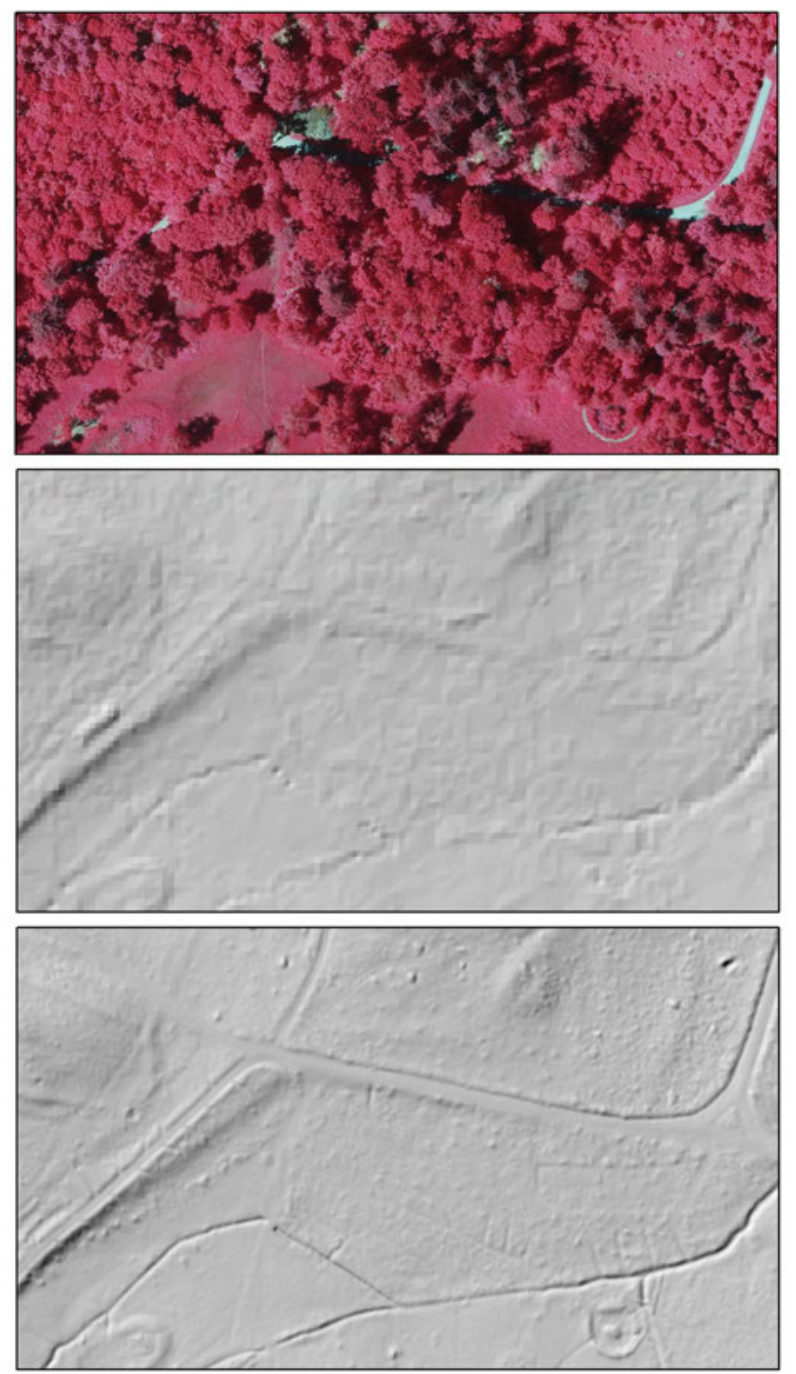

0

50 $100 \mathrm{~m}$

(

Abb 5 Steigerung des Detaillierungsgrades des modellierten Bodenmodells mit höherer Punktdichte an einem Beispiel im Kanton Zürich. Oben: FIR-Orthobild 2014 (৫ Kanton Zürich). Mitte: Reliefdarstellung des DTM, berechnet aus den ALS-Daten der Befliegung 2002. Unten: Reliefdarstellung des DTM, berechnet aus den kantonalen ALS-Daten der Befliegung 2014.

sich aus den hohen Punktdichten ergeben, können in den gebräuchlichen GIS-Umgebungen problemlos bewältigt werden und erlauben somit eine Integration in gewohnte Arbeitsabläufe. Mit Werkzeugen wie zum Beispiel FUSION ${ }^{1}$ oder LAStools ${ }^{2}$ lassen sich auch sehr grosse Datensätze verwalten und auswerten.

\section{Verbesserungen bei der Datenerfassung vor dem Laubaustrieb}

Meist werden ALS-Daten nach der Schneeschmelze und vor dem Laubaustrieb aufgenommen (unbelaubter Zustand, sog. Leaf-off-Daten), um vor allem den Boden unter der Vegetation optimal modellieren zu können. Bis vor wenigen Jahren eigneten sich diese Daten für die Modellierung der Struktur von Laubwald nur bedingt. Die Punktdichte mit zum Teil weniger als einem Punkt pro Quadratme- ter war gering, und durch das Fehlen der Blätter wurden wenige Reflexionen auf oder innerhalb der Vegetation aufgezeichnet. So fehlen zum Beispiel im landesweiten ALS-Datensatz von 2001 der Swisstopo in weiten Teilen des laubwaldreichen Kantons BaselLandschaft praktisch alle Laubbäume. Durch die deutliche Erhöhung der Punktdichte in den letzten zehn Jahren hat sich die Verwendbarkeit der Leafoff-Daten für Analysen im Waldbereich deutlich verbessert (Abbildung 6). White et al (2015) konnten in einer Arbeit in Kanada zeigen, dass die Modellierung von forstlich relevanten Parametern (z.B. Volumen, Biomasse, Grundfläche) ohne Weiteres auch mit ALS-Daten vor dem Blattaustrieb durchgeführt werden kann. Die Unterschiede zu Aufnahmen im belaubten Zustand waren sehr gering. Im Kanton Aargau wurden 2014 zwei ALS-Befliegungen, eine im März (Leaf-off) und eine im Juli (belaubter Zustand; Leaf-on), durchgeführt. Basierend auf diesen Aufnahmen wurden zwei Vorratsmodellierungen durch Verknüpfung der ALS-Daten mit Daten der terrestrischen Stichprobeninventur des Kantons vorgenommen. Beide Modelle waren nicht zufriedenstellend, da der Zeitabstand zwischen der terrestrischen Aufnahme und den ALS-Befliegungen sehr gross war (neun Jahre) und die genaue Lage der Probeflächen nicht bekannt ist. Die Modelle glichen sich aber stark. Auch zukünftige kantonale und nationale ALS-Befliegungen werden mit grosser Wahrscheinlichkeit zumeist in der laublosen Zeit erfolgen, da die exakte Modellierung des Bodens für ein aktuelles DTM eine hohe Priorität hat. Aktuelle Forschungsresultate zeigen nun aber die Eignung dieser Daten auch für forstliche Fragestellungen und Anwendungen (White et al 2015).

\section{Entwicklungen bei Scannern mit Potenzial für Waldanalysen}

Praktische Anwendungen beschränken sich heute auf die räumliche Verteilung der diskreten ALS-Punkte. Obwohl FWF-Scanner die volle Information der Rückstrahlung bereits aufzeichnen, wird diese Information in der Praxis (noch) nicht verwendet. Mit der Echoweite der Reflexionen - eine Information, die aus den FWF-Daten gerechnet werden kann - lässt sich beispielsweise die Qualität der DTM im Wald verbessern. Auch wurden mithilfe der Echoweite gute Ergebnisse bei der Differenzierung von Nadel- und Laubbäumen sowie bei der Erkennung der Lärche erzielt (Hollaus et al 2009). Auch die Form der Rückstrahlung wird für die Baumartenunterscheidung untersucht (Bruggisser 2016). Es sind aber noch keine praxisnahen Werkzeuge zur Bearbeitung dieser Informationen verfügbar, weshalb sie

\footnotetext{
$1 \mathrm{http}: / /$ forsys.cfr.washington.edu/fusion/fusionlatest.html (16.3.2017)

2 https://rapidlasso.com/ (16.3.2017)
} 

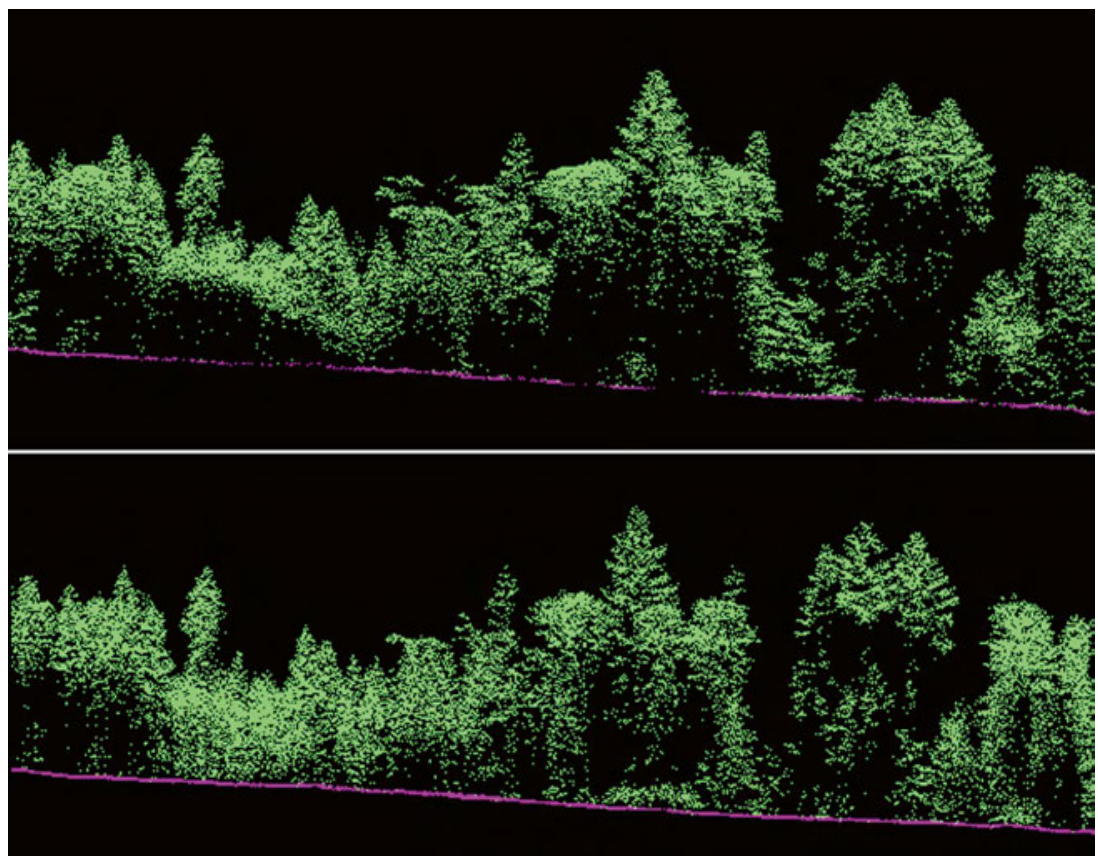

Abb 6 Profil durch die ALS-Punktwolke im Kanton Aargau. Oben: Leaf-on-Sommerbefliegung. Unten: Leaf-off-Frühjahrsbefliegung. Das Kronendach wird sehr ähnlich wiedergegeben. Allerdings tritt die Unterschicht bei den Leaf-off-Daten deutlicher hervor, da mehr Lichtpulse durch das blattlose Kronendach dringen können.

auch nicht auf grosser Fläche genutzt werden können. Eine Kombination von 3-D-Erfassung und spektraler Information stellen multispektrale Laserscanner in Aussicht. Dabei werden Lichtpulse mit verschiedenen Wellenlängen gesendet, um zum Beispiel in einer Flugkampagne Bodendaten im flachen Wasser und am trockenen Boden zeitgleich zu erfassen. Für Vegetationsanalysen könnten aus den Wellenlängen im Rot- und im Nahinfrarot-Bereich Vegetationsindizes aus den 3-D-Punkten gerechnet werden. So könnten Punkte auf Blättern und Ästen unterschieden werden. Erste multispektrale Laserscanner existieren bereits in der Praxis (z.B. Optech Titan). Allerdings lassen sich die Reflexionen aus verschiedenen Quellen noch nicht einwandfrei in Übereinstimmung bringen, weshalb diese Systeme für den Praxiseinsatz noch nicht bereit sind.
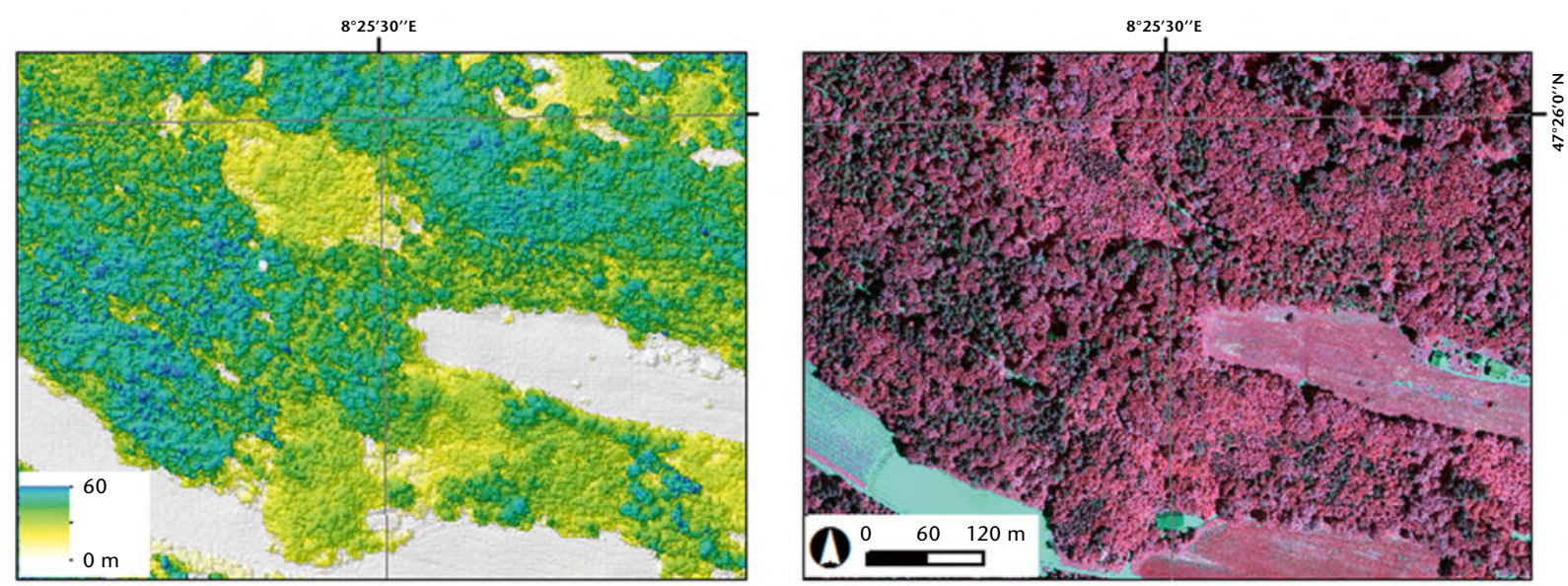

Abb 7 Aus Stereoluftbildern berechnete Vegetationshöhen (links). Als Vergleich ist rechts das entsprechende FIR-Orthofoto abgebildet. ๑ Landesforstinventar

\section{3-D-Information aus digitaler Bildkorrelation}

Nicht immer ist ein aktueller ALS-Datensatz verfügbar. Nach den landesweiten Befliegungen der Schweiz zwischen 2001 und 2008 war keine Nachführung geplant. Einzelne Kantone führten daher seit 2012 eigene ALS-Befliegungen durch, manche mit und andere ohne Nachführungskonzept. Der Bestand der ALS-Daten über die Schweiz ist heute daher sehr heterogen. Für die aktuelle Beschreibung der Oberfläche von Wäldern ist das ein Problem. Es kann behoben werden durch die sogenannte digitale Bildkorrelation.

Aus stereoskopischen Aufnahmen kann die dritte Dimension, die Höhe, berechnet werden. Diese Methode wird seit Jahrzehnten angewendet. Sie wurde allerdings erst durch neue Algorithmen wie das Semi Global Matching (SGM) unter dem Begriff «Dense Image Matching» wieder populär. Bei dieser Methode werden aus den überlappenden Bildern sehr dichte Punktwolken gerechnet, zum Teil mit einer höheren Dichte als beim ALS. Der grosse Unterschied zum ALS ist jedoch, dass 3-D-Informationen nur zur Oberfläche von Vegetation, nicht aber zu ihrem Innern oder zum darunter liegenden Boden generiert werden. Für ein nDOM muss daher ein DTM aus einer ALS-Befliegung vorliegen - nur dann können Baum- oder Bestandeshöhen berechnet werden (Abbildung 7). Der grosse Vorteil der Bildkorrelation ist, dass Stereobilder aus Luftbildbefliegungen sehr oft und in sehr hoher zeitlicher Dichte vorhanden sind. Sind solche Bilder verfügbar, so kann mit vertretbarem Aufwand und ohne zusätzliche Flugkampagne ein DOM mit hoher räumlicher Auflösung gerechnet werden. Wichtige forstliche Merkmale (Volumen, Stammzahl, Grundfläche) können mit einer ähnlichen Genauigkeit modelliert werden wie mit ALS (Järnstedt et al 2012, Straub et al 2013). Vor allem flächenbasierte Modelle (z.B. auf Bestandesebene) funktionieren mit Bildkorrelation so gut wie mit ALS. 
Bei der Detektion von Einzelbäumen ist ALS der Bildkorrelation mit Stereobildern noch überlegen, vor allem wenn für die Korrelation Bildmaterial aus den Standardbefliegungen für Orthobilder verwendet wird. Mit Stereobildern lassen sich aber grossflächig sehr effizient 3-D-Informationen generieren. Für die Schweiz steht seit 2015 ein detailliertes nationales VHM mit einer räumlichen Auflösung von $1 \mathrm{~m}$, gerechnet aus den digitalen ADS80-Luftbildern, zur Verfügung (Ginzler \& Hobi 2016). Auch in anderen Ländern (z.B. Österreich, Deutschland) sind Arbeiten zu landesweiten Oberflächenmodellen aus Bildkorrelation am Laufen.

\section{Terrestrisches Laserscanning}

In den letzten Jahren sind viele Forschungsarbeiten zum Thema Anwendung und Möglichkeiten von terrestrischem Laserscanning (TLS) in Waldinventuren durchgeführt worden. Die Technik bei TLS ist ähnlich wie bei ALS. Der Sensor ist meist auf einem Stativ befestigt und erlaubt eine Messung von horizontal $360^{\circ}$ und vertikal $320^{\circ}$ (vom Zenit bis fast senkrecht auf den Boden, nur unter dem Scanner kann nicht gemessen werden). Die Flächenleistung von TLS ist deutlich geringer als jene flugzeuggetragener Sensoren. Meist sind die Aufnahmen auf Flächen von wenigen Aren beschränkt. Ein potenzielles Einsatzgebiet ist die Aufnahme von Stichprobenplots einer Inventur. Die Messungen werden sehr effizient durchgeführt, und eine Reihe von inven-

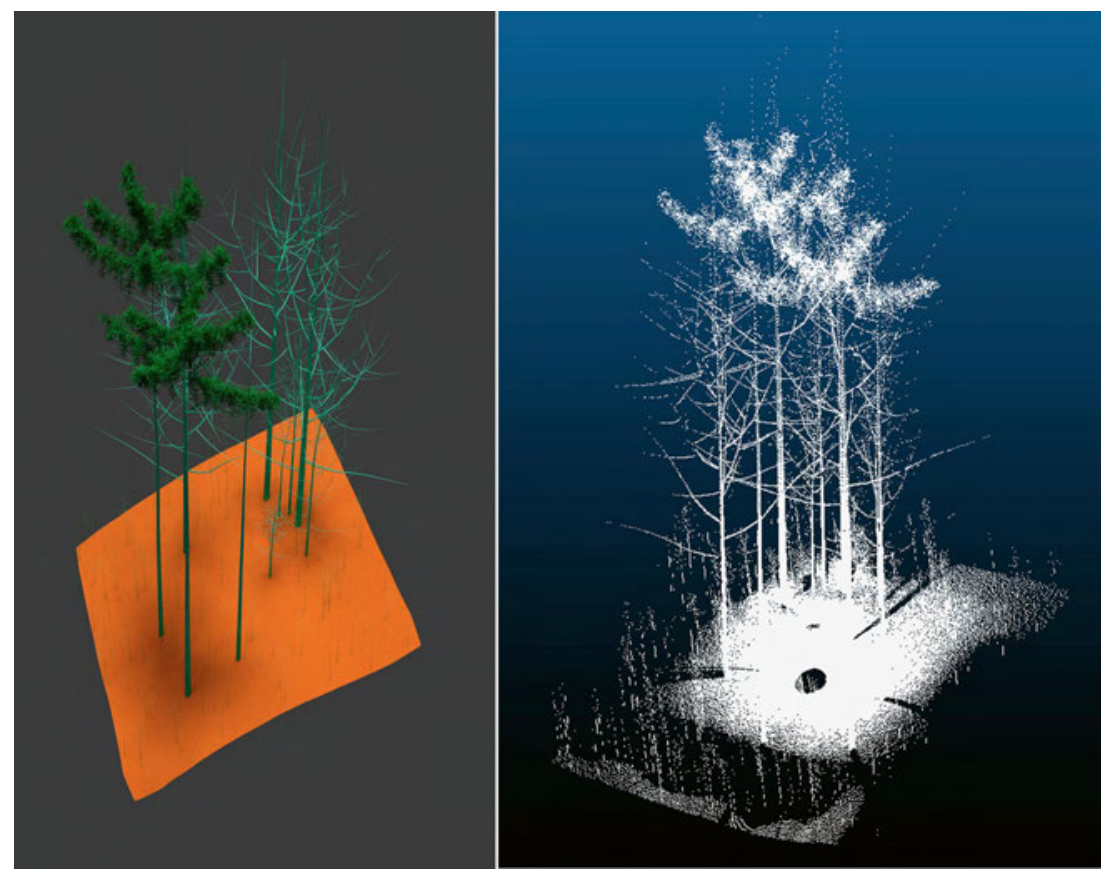

Abb 8 Zur Analyse, wie der Scanner auf den Probeflächen platziert werden muss, damit möglichst keine Abschattungen entstehen, laufen im Landesforstinventar (LFI) derzeit Tests am Computer mit synthetischen Probeflächen und virtuellen Scans. Links: synthetische Probefläche, die Bäume wurden auf Basis von LFI-Felddaten generiert. Rechts: virtueller Scan einer synthetischen Probefläche. Abbildungen: Meinrad Abegg turrelevanten Merkmalen kann aus den Millionen von aufgezeichneten 3-D-Punkten berechnet werden (Vastaranta et al 2009, Vonderach et al 2012).

Es gibt verschiedene Aufnahmetechniken: Beim «Single Scan» wird am Probeflächenzentrum ein einziger Scan durchgeführt. Der Zeitaufwand ist gering, und aus dem Scan können Bäume identifiziert und Baummerkmale abgeleitet werden (Astrup et al 2014). Die Punktdichte liegt üblicherweise im Bereich von 1-2 $\mathrm{cm}$ bei einem Abstand von $10 \mathrm{~m}$ vom Scanner. Der Nachteil beim Single Scan ist die Abschattung von Bäumen in Abhängigkeit von der Entfernung. Je weiter ein Baum vom Scanner entfernt ist, desto grösser wird die Wahrscheinlichkeit, dass der Baum von einem anderen verdeckt wird. Die Bäume auf der Fläche werden auch nur an der dem Scanner zugewandten Seite abgetastet, ihre Rückseite wird nicht erfasst.

Eine Variante der Single-Scan-Technik ist der «Multi Single Scan». Dabei wird der Scanner auf einer Probefläche an mehreren Orten aufgestellt, und es wird jeweils ein Einzelscan durchgeführt. Anhand der detektierten gemeinsamen Bäume in jedem Scan können die Einzelscans zu einem Datensatz verknüpft werden. Dadurch wird die Abschattung deutlich reduziert, und die Bäume können von allen Seiten erfasst werden. Die aufwendigste, aber auch genaueste Variante ist der «Multiscan». Dabei werden ebenfalls mehrere Scannerstandorte verwendet, die Verknüpfung der Scans erfolgt aber mit mithilfe von Referenzobjekten, meist Kugeln, die eigens platziert werden. Im Architekturscan, also bei Gebäuden, funktioniert die automatische Verknüpfung auch bereits ohne solche Referenzobjekte, im Wald jedoch noch nicht.

Bei der Berechnung von Merkmalen auf PlotEbene aus TLS-Daten müssen zuerst die Anzahl und die Position von möglichst vielen Einzelbäumen auf dem Plot berechnet werden. Dies geschieht meist durch eine Einpassung von Kreisen auf den Scanpunkten in der Höhe von $1.30 \mathrm{~m}$ ab Boden (z.B. Lindberg et al 2012). So erhält man neben der Anzahl und der Position der Bäume auch deren BHD. Die Detektionsrate von Einzelbäumen ist stark von der Stammzahl pro Hektare sowie der Waldstruktur abhängig. Bei Stammzahlen von 200 bis 400 Stämmen/Hektare sind Erkennungsraten von bis zu $80 \%$ möglich, bei höheren Stammzahlen sinkt die Erkennungsrate schnell auf unter 50\% (Liang et al 2016). Die Genauigkeit der BHD-Berechnung variiert je nach Waldtyp und Scanmethoden stark, die Abweichungen reichen von weniger als $1 \mathrm{~cm}$ bis etwa 3-4 cm (Liang et al 2016). Im Rahmen des LFI sind aktuell Arbeiten zu Detektionsraten und ableitbaren Baummerkmalen auf Stichprobenflächen am Laufen (Abbildung 8).

Grosses Potenzial liegt in der Ermittlung von Durchmessern auf verschiedenen Höhen, da die Be- 
rechnungen nicht auf die BHD-Höhe beschränkt sind. Entsprechend dürfen Verbesserungen bei den Modellen zur Ermittlung des Holzvolumens erwartet werden. Aber auch die Stammqualität, insbesondere die Schaftform und die Länge des astfreien Schaftes, dürfte bereits in naher Zukunft besser erfasst werden können. Forschungsarbeiten laufen aktuell im LFI auch zur Quantifizierung der Verjüngung sowie von Mikrohabitaten an Stämmen mittels TLS.

\section{Fazit und Ausblick}

Abschliessend kann festgehalten werden, dass sich prinzipiell sowohl die neueren flugzeuggetragenen Sensoren in ihrer ganzen Vielfalt als auch die Satellitensensoren für forstliche Anwendungen in der Schweiz eignen. Dank regelmässiger - oft landesweiter - Befliegungskampagnen haben digitale Luftbilder weiter an Attraktivität gewonnen. Die zeitliche Auflösung ist sehr hoch, und die 3-D-Informationen dieser Bilder haben grosses Potenzial für die Beschreibung wichtiger Waldattribute. Die räumliche Auflösung wird sich weiter verbessern. So werden die Luftbilddaten von Swisstopo in Zukunft eine Auflösung von $10 \mathrm{~cm}$ aufweisen. ALS-Daten werden vermehrt verfügbar sein. Swisstopo plant eine neue landesweite ALS-Befliegung bis zur Baumgrenze. Eine regelmässige Nachführung wie bei den Luftbildern ist allerdings nicht geplant. Unbemannte Flugobjekte (unmanned aerial vehicles, UAV) werden in Zukunft eine wichtige Rolle spielen. Die Sensoren für UAV werden kleiner und leichter werden. ALSAufnahmen mit UAV sind bereits heute möglich und werden in Zukunft auch grössere Gebiete abdecken können. Der nächste Schritt wird der Einsatz von UAV unter dem Kronendach sein - erste Forschungsarbeiten dazu sind bereits im Gang (Chisholm et al 2013).

Haben nun die klassischen terrestrischen Inventuren und Messungen im Wald ausgedient? Die Antwort auf diese Frage ist ein klares Nein. Fernerkundung ist ein wunderbares Werkzeug, um Informationen zu forstlichen Merkmalen in hohem Detaillierungsgrad und über grosse Flächen zu erhalten. Manche Merkmale, zum Beispiel Höhen, können mit Methoden der Fernerkundung schneller und vielleicht auch genauer bestimmt werden. Andere Merkmale, zum Beispiel Baumarten, speziell seltene Baumarten, stellen die Fernerkundung noch immer vor grosse Herausforderungen. Ist man sich der Möglichkeiten, aber auch der aktuellen Grenzen der Fernerkundung bewusst, so lassen sich sehr viele spannende und nützliche Produkte und Anwendungen für den Waldbereich realisieren.

Eingereicht: 25. Oktober 2016, akzeptiert (mit Review): 27. März 2017

\section{Literatur}

ASTRUP R, DUCEY MJ, GRANHUS A, RITTER T, VON LÜPKE N (2014) Approaches for estimating stand-level volume using terrestrial laser scanning in a single-scan mode. Can J For Res 44: 666-676.

BRUGGISSER M (2016) Retrieval of higher order statistical moments from full-waveform LiDAR data for tree species classification. Zurich: Univ Zurich, Master Thesis. 75 p.

CHISHOLM R, CUI J, LUM S, CHEN B (2013) UAV LiDAR for belowcanopy forest surveys. J Unman Veh Syst 1: 61-68.

DYMYTROVA L, STOFER S, GINZLER C, BREINER F, SCHEIDEGGER $C$ (2016): Forest-structure data improve distribution models of threatened habitat specialists: Implications for conservation of epiphytic lichens in forest landscapes. Biol Conserv 196: 31-38.

FASSNACHT FE, NEUMANN C, FÖRSTER M, BUDDENBAUM H, GHOSH A ET AL (2014) Comparison of feature reduction algorithms for classifying tree species with hyperspectral data on three central European test sites. IEEE J Sel Top Appl Earth Obs Remote Sens 7: 2547-2561.

GILLIS M, LECKIE D (1996) Forest inventory update in Canada. For Chron 72: 138-156.

GINZLER C, HOBI ML (2016) Das aktuelle Vegetationshöhenmodell der Schweiz: spezifische Anwendungen im Waldbereich. Schweiz Z Forstwes 167: 128-135. doi: 10.3188/szf.2016.0128

HANSEN MC, POTAPOV PV, MOORE R, HANCHER M, TURUBANOVA SA ET AL (2013) High-resolution global maps of 21 st-century forest cover change. Science 342: 850-853.

HILL A, BRESCHAN J, MANDALLAZ D (2014) Accuracy assessment of timber volume maps using forest inventory data and LiDAR canopy height models. Forests 5: 2253-2275.

HOLLAUS M, MÜCKE W, HÖFLE B, DORIGO W, PFEIFER N ET AL (2009) Tree species classification based on full-waveform airborne laser scanning data. In: Proc 9th International Conference on LiDAR Applications for Assessing Forest Ecosystems (Silvilaser 2009), 14-16 Oct 2009, College Station, TX, USA. pp. 54-62.

HOLLAUS M, PFEIFER N (2017) Multitemporales, flugzeuggetragenes Laserscanning für die Forstwirtschaft. Schweiz Z Forstwes 168: 134-141. doi: 10.3188/szf.2017.0134

HUBER N, KIENAST F, GINZLER C, PASINELLI G (2016) Using remote-sensing data to assess habitat selection of a declining passerine at two spatial scales. Landsc Ecol 31: 1919-1937.

HUBER S, KNEUBÜHLER M, PSOMAS A, ITTEN K, ZIMMERMANN NE (2008) Estimating foliar biochemistry from hyperspectral data in mixed forest canopy. For Ecol Manage 256: 491-501.

IMMITZER M, ATZBERGER C, KOUKAL T (2012) Tree species classification with Random Forest using very high spatial resolution 8-Band WorldView-2 satellite data. Remote Sens 4: 2661-2693.

JÄRNSTEDT J, PEKKARINEN A, TUOMINEN S, GINZLER C, HOLOPAINEN M (2012) Forest variable estimation using a high resolution digital surface model. ISPRS J Photogram Remote Sens 74: 78-84.

LAUSCH A, HEURICH M, GORDALLA D, DOBNER HJ, GWILLYMMARGIANTO S ET AL (2013) Forecasting potential bark beetle outbreaks based on spruce forest vitality using hyperspectral remote-sensing techniques at different scales. For Ecol Manage 308: 76-89.

LEITERER R, TORABZADEH H, FURRER R, SCHAEPMAN ME, MORSDORF F (2015) Towards automated characterization of canopy layering in mixed temperate forests using airborne laser scanning. Forests 6: 4146-4167.

LIANG X, KANKARE V, HYYPPÄ J, WANG Y, KUKKO A ET AL (2016) Terrestrial laser scanning in forest inventories. ISPRS J Photogram Remote Sens 115: 63-77. 
LINDBERG E, HOLMGREN J, OLOFSSON K, OLSSON H (2012) Estimation of stem attributes using a combination of terrestrial and airborne laser scanning. Eur J For Res 131: 1917-1931.

MENK J, DORREN L, HEINZEL J, MARTY M, HUBER M (2017) Evaluation automatischer Einzelbaumerkennung aus luftgestützten Laserscanning-Daten. Schweiz Z Forstwes 168: 151-159. doi: 10.3188/szf.2017.0151

NILSSON M, NORDKVIST K, JONZÉN J, LINDGREN N, AXENSTEIN P ET AL (2016) A nationwide forest attribute map of Sweden predicted using airborne laser scanning data and field data from the National Forest Inventory. Remote Sens Environ. http://dx.doi.org/10.1016/j.rse.2016.10.022

OESTER B (1992) Flächendeckende Infrarot-Luftbilder zur Erfassung der Waldschäden. Birmensdorf: Eidgenöss Forsch.anst WSL, Ber 334. pp. 18-23.

SCHERRER HU, GAUTSCHI H, HAUENSTEIN P (1990) Flächenhafte Waldschadeninventur mit Infrarot-Luftbildern 1:9000. Schlussbericht Programm Sanasilva 1984-1987, Teilprogramm 3. Birmensdorf: Eidgenöss Forsch.anst WSL, Ber 318. $101 \mathrm{p}$.

STRAUB C, WEINACKER H, KOCH B (2010) A comparison of different methods for forest resource estimation using information from airborne laser scanning and CIR orthophotos. Eur J For Res 129: 1069-1080.

STRAUB C, STEPPER C, SEITZ R, WASER LT (2013) Potential of UltraCamX stereo images for estimating timber volume and basal area at the plot level in mixed European forests. Can J For Res 43: 731-741.

\section{Développements dans le domaine de la télédétection pour les usages forestiers}

Au cours de la dernière décennie, de grands progrès ont été faits dans le domaine des méthodes et des données de la télédétection pour les usages forestiers. Beaucoup de jeux de données sont disponibles non seulement pour les études de cas et le développement de méthodes, mais aussi pour des utilisations à grande échelle, et elles sont maintenant fréquemment actualisées. Des photos aériennes - aujourd'hui également en proche infrarouge - sont devenues standard par l'emploi de caméras numériques. Les orthophotos infrarouge couleur peuvent facilement être intégrées dans les applications SIG aux niveaux cantonal et fédéral. Les données de scanner laser aéroporté sont presque devenues un standard pour beaucoup d'applications. Des outils intégrés dans les environnements numériques courants permettent une analyse très efficace des nuages de points $3 \mathrm{D}$ et la réalisation de produits utiles au domaine forestier. Le scanner laser terrestre est presque opérationnel. La télédétection devient de plus en plus un outil pratique pour le travail quotidien. Tout en restant conscient de ses potentiels, mais aussi de ses limites, la télédétection peut amener des contributions substantielles pour appuyer et optimiser les inventaires terrestres et générer une plus-value à grande échelle.
VASTARANTA M, MELKAS T, HOLOPAINEN M, KAARTINEN H, HYYPPÄ J ET AL (2009) Laser-based field measurements in treelevel forest data acquisition. Photogramm J Finland 21: 51-61.

VONDERACH C, VÖGTLE T, ADLER P, NORRA S (2012) Terrestria laser scanning for estimating urban tree volume and carbon content. Int J Remote Sens 33: 6652-6667.

WASER LT (2013) Stand und Perspektiven einer landesweiten Baumartenklassifikation mit digitalen Luftbildern. Schweiz Z Forstwes 164: 95-103. doi: 10.3188/szf.2013.0095

WASER LT, KÜCHLER M, JÜTTE K, STAMPFER T (2014) Evaluating the potential of World-View-2 data to classify tree species and different levels of ash mortality. Remote Sens 6: 4515-4545.

WHITE JC, ARNETT J, WULDER M, TOMPALSKI P, COOPS N (2015) Evaluating the impact of leaf-on and leaf-off airborne laser scanning data on the estimation of forest inventory attributes with the area based approach. Can J For Res 45: 1498-1513.

ZELLWEGER F, BALTENSWEILER A, GINZLER C, ROTH T, BRAUNISCH V ET AL (2016) Environmental predictors of species richness in forest landscapes: abiotic factors versus vegetation structure. J Biogeogr 43: 1080-1090.

ZELLWEGER F, BOLLMANN K (2017) Der Schweizer Wald und seine Biodiversität: LiDAR ermöglicht neue Waldstrukturanalysen. Schweiz Z Forstwes 168: 142-150. doi: 10.3188/szf.2017.0142

\section{Progress in remote sensing for forestry applications}

Over the past ten years significant advances have been made in remote sensing data and methods for forestry applications. In many cases datasets are no longer limited to use for case studies or the development of methods, but are available for large area applications, often with high temporal resolution. Aerial image acquisition, including in near infrared, has become standard thanks to the use of digital cameras. Colour infrared orthophotos are easily embedded into GIS applications at the cantonal and national level. Aerial laser scanning data has almost become the norm for many applications. Tools integrated into common working environments are available which allow for the efficient analysis of 3-D point clouds and the realisation of valuable products describing forested areas. Terrestrial laser scanning is also nearing operational applicability for many purposes. Remote sensing is continually developing into a practical instrument for daily work. As long as users are aware of the possibilities and also the limitations, remote sensing offers substantial potential to support and optimise terrestrial inventory and for the generation of additional large-scale values. 